\title{
Chromosome specific comparative genome hybridisation for determining the origin of intrachromosomal duplications
}

\author{
Darren K Griffin, Despina Sanoudou, Emma Adamski, Christine McGiffert,
} Patricia O'Brien, Johannes Wienberg, Malcolm A Ferguson-Smith

\begin{abstract}
Chromosome specific comparative genome hybridisation (CGH) is a novel approach for the detection of cytogenetic abnormalities. It combines flow sorting of chromosomes, degenerate oligonucleotide primed (DOP)-PCR and a modified comparative genome hybridisation (CGH) technique to define the site and extent of intrachromosomal duplications. Chromosome specific paint probes for aberrant chromosomes and their normal homologues from four subjects with unbalanced duplications within chromosomes 2p1115, 3q25-26, 5q34-qter, and 12q23-24.2 were made. They were then cohybridised on normal metaphase spreads and the ratio of their relative intensities of hybridisation analysed. The results were compared to those of similar experiments where regular CGH was performed on the same four patients. We provide evidence that this method can detect duplications and deficiencies which might be missed by conventional CGH, as the ratio of hybridisation of abnormal/normal DNA is $2: 1$ rather than 3:2. It is the method of choice where mosaicism is present or where only one of several homologous chromosomes is duplicated. Furthermore, it suggests that DOP-PCR amplifies all or most of the euchromatic regions of the genome equally.

$(\mathcal{O}$ Med Genet 1998;35:37-41)
\end{abstract}

Keywords: CGH; reverse chromosome painting; duplication; DOP-PCR

Reverse chromosome painting is an approach for determining the nature of structural chromosome aberrations including those inaccessible to $G$ banding. That is, in cases where results from $G$ banding are unclear, chromosome paints can be made from abnormal chromosomes and used as in situ hybridisation probes onto normal metaphases. ${ }^{1}$ The procedure involves chromosome sorting by flow cytometry and the identification and sorting of aberrant peaks on a flow karyotype with subsequent amplification and labelling by degenerate oligonucleotide primed (DOP)-PCR. ${ }^{2} \mathrm{Al}-$ though invaluable for locating deletions and rearrangements between different chromosomes, it is not adequate for determining the origin of intrachromosomal duplications. For example, a paint made from a deleted chromo- some will hybridise to its complementary normal chromosome except in the deleted region, but a paint made from a chromosome with an intrachromosomal duplication will hybridise only to the complementary normal chromosome and nowhere else on the karyotype. To circumvent this problem, we developed a novel approach which involves simultaneous hybridisation of the duplicated chromosome and its normal homologue using dual colour fluorescent in situ hybridisation. The paint derived from the duplicated chromosome hybridises more strongly in the duplicated region than in its homologue. This takes advantage of a relative over-representation of probe DNA producing a stronger signal in the duplicated region. Using this approach, small intrachromosomal duplications were detected easily using image processing software designed for comparative genome hybridisation (CGH). This novel approach is similar to $\mathrm{CGH}^{3}$ in that it measures the relative hybridisation of probe DNA along the chromosomal axis; however, in this case, the analysis is performed on one chromosome pair alone.

\section{Methods}

In a preliminary series, four patients were investigated for cytogenetic abnormalities at the Clinical Cytogenetic Laboratory, East Anglian Genetics Service, Addenbrooke's NHS Trust, Cambridge, UK. Classical G banding analysis showed that each had a chromosomal duplication, but the origin of the duplication could not be accurately determined. Patient 1 had a duplication of the short arm of chromosome 2 apparently originating from the centromeric region, patient 2 had a duplication in the long arm of chromosome 3 apparently originating from around band $3 q 25$, patient 3 had a duplication of the terminal portion of the long arm of chromosome 5, and patient 4 had a duplication of the long arm of chromosome 12 originating from around band $12 \mathrm{q} 24$. Thus, in each case, the aberrant chromosome was identified by $G$ banding before flow sorting.

Chromosome paints from each patient were generated as follows. Chromosomes were prepared for flow sorting as described previously, ${ }^{1}$ spun briefly (100 $g$ for one minute to remove any debris), then the supernatant stained with $2 \mu \mathrm{g} / \mathrm{ml}$ Hoechst 33258 (Sigma) and $40 \mu \mathrm{g}$ Chromomycin A3 (Sigma). Bivariate flow karyotypes were generated on a FACStar Plus (Beckton Dickinson) dual laser flow 

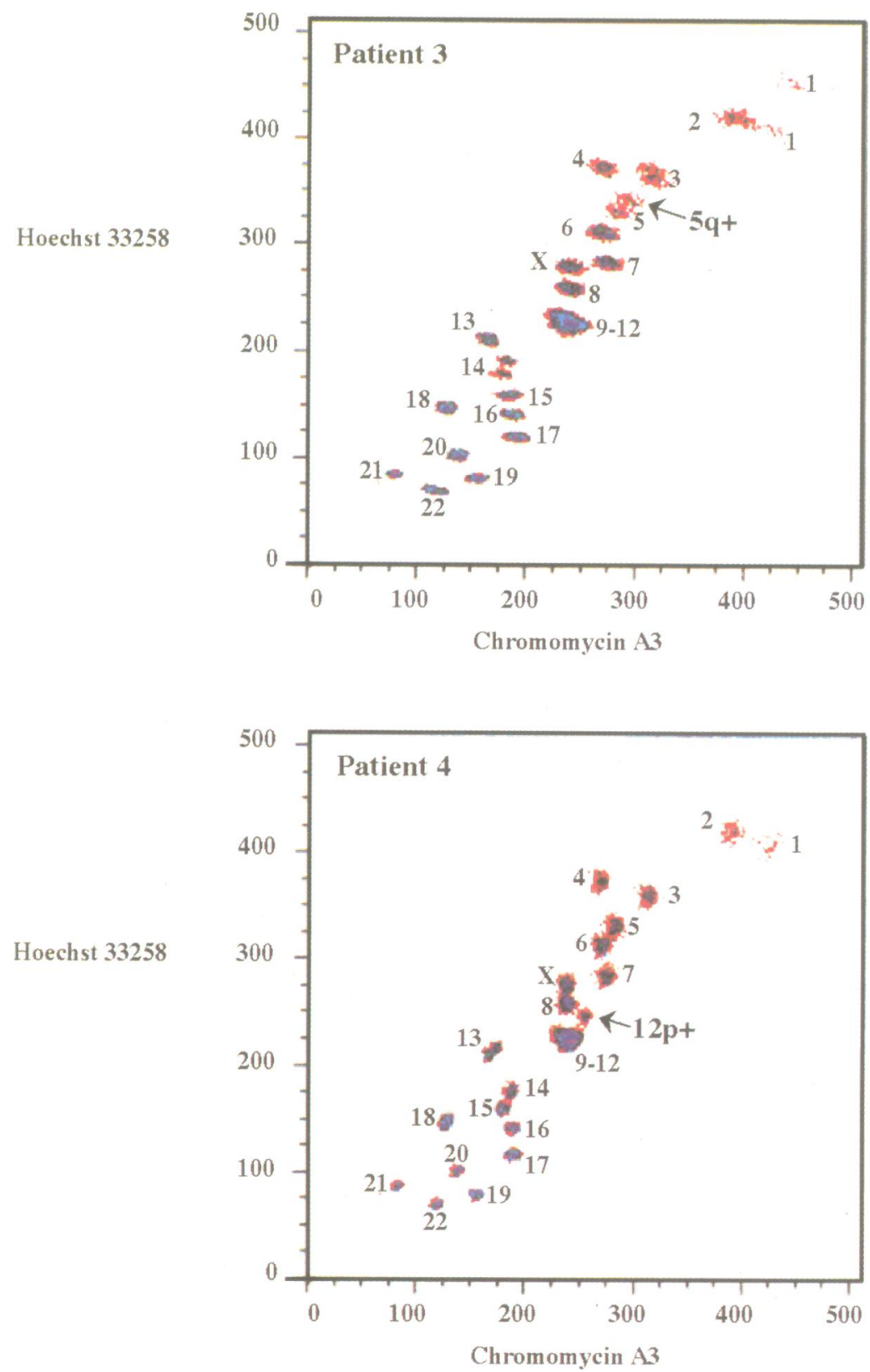

Figure 1 Flow karyotypes of patients 3 and 4. The aberrant peaks are clearly shown.

cytometer equipped with two $5 \mathrm{~W}$ argon ion lasers. Fig 1 shows the bivariate flow karyotypes for patients 3 and 4 . The aberrant chromosome was identified on the flow karyotype and approximately 400 chromosomes from it and, where appropriate, from the corresponding normal peak were flow sorted into a $0.5 \mathrm{ml}$ Eppendorf tube containing water. A primary round of DOP-PCR amplification was performed on these chromosomes. ${ }^{12}$ From each of these reactions $1 \mu \mathrm{l}$ was used as a template for a secondary DOP-PCR amplification incorporating biotin-dUTP. This facilitated amplifica- tion and labelling of the aberrant chromosome, thus making a chromosome paint. ${ }^{12}$ Single colour hybridisation experiments to normal human metaphases by reverse chromosome painting determined that each duplication was intrachromosomal. For patients 1, 2, and 3, the normal homologue was also flow sorted and labelled using FITC-dUTP or digoxigenin dUTP in the same way as described. For patient 4 , a single chromosome paint could not be made from the normal homologue, since a normal chromosome 12 sorts in the same peak as chromosomes 9,10 , and 11 in the human karyotype. Therefore, a commercially available fluorescein labelled chromosome 12 paint (Cambio) was used. Biotin labelled paints from duplicated chromosomes were cohybridised with normal paints labelled with FITC or digoxigenin on metaphases made from a normal male lymphoblastoid cell line. Briefly, chromosomes were prepared by standard procedures, dehydrated, then aged overnight at $37^{\circ} \mathrm{C}$. A one minute 30 second denaturation of chromosomal DNA $\left(65^{\circ} \mathrm{C}\right.$ in $70 \%$ formamide, $2 \times$ SSC) preceded immediate plunging in ice cold $70 \%$ ethanol, dehydration, and air drying. Meanwhile, the chromosome paints were denatured at $65^{\circ} \mathrm{C}$ for 10 minutes, then preannealed at $37^{\circ} \mathrm{C}$ for 30 minutes in standard hybridisation buffer (containing 50\% formamide, $10 \%$ dextran sulphate, and cot 1 DNA). Following a three day hybridisation and relevant posthybridisation washes $(3 \times 10 \mathrm{~min}$ utes $50 \%$ formamide, $2 \times$ SSC, $2 \times 10$ minutes $2 \times \mathrm{SSC}, 1 \times 10$ minutes $0.5 \times \mathrm{SSC}, 45^{\circ} \mathrm{C}$ ), biotinylated probes were detected with $\mathrm{Cy} 3$ avidin ( $1: 500$ dilution in $4 \times$ SSC, $0.1 \%$ Tween $20,1 \% \mathrm{BSA}$ ), digoxigenin labelled paints with FITC conjugated antidigoxigenin (1:200 dilution), and fluoresceinated paints were amplified using sequential layers of rabbit antiFITC (1:200 dilution) and FITC conjugated goat antirabbit antibody (1:500 dilution). Finally, chromosomes were counterstained with DAPI and mounted in "Citifluor" antifade medium before viewing.

In order to compare this new technique with the already established comparative genomic hybridisation (CGH) procedure, genomic DNA was extracted from each of these patients and then amplified and labelled with biotin using two rounds of DOP-PCR as described above. Comparative genomic hybridisation was performed using the same protocol as described above using DOP-PCR labelled genomic DNA from a karyotypically normal subject as a reference standard. Furthermore, in order to make a fair comparison between the two techniques, experiments were performed on chromosomes from the same cell line in both instances.

Table 1 Tandem duplications using CGH

\begin{tabular}{|c|c|c|c|c|c|}
\hline Patient & $\begin{array}{l}G \text { band analysis (band location of } \\
\text { extra material) }\end{array}$ & $\begin{array}{l}\text { Chromosome specific } \\
\text { CGH }\end{array}$ & $C G H$ result & Nature of duplication & $\begin{array}{l}\text { Approx size of } \\
\text { duplication }\end{array}$ \\
\hline $\begin{array}{l}1 \\
2 \\
3 \\
4\end{array}$ & $\begin{array}{l}46, X Y, 2 p+(p 11-p 22) \\
46, X Y, 3 q+(q 25) \\
46, X X, 5 q+(q 34) \\
46, X X, 12 q+(q 24)\end{array}$ & $\begin{array}{l}\text { dup 2p11-22 } \\
\text { dup 3q25-26.1 } \\
\text { dup 5q34-qter } \\
\text { dup 12q23-24.2 }\end{array}$ & $\begin{array}{l}\text { dup 2p11-31 } \\
\text { Not detected } \\
\text { Not detected } \\
\text { dup 12q23-qter } \\
\text { (peak at pter ignored) }\end{array}$ & $\begin{array}{l}\text { Tandem intrachromosomal } \\
\text { Tandem intrachromosomal } \\
\text { Tandem intrachromosomal } \\
\text { Tandem intrachromosomal }\end{array}$ & $\begin{array}{l}\sim 65 \mathrm{Mb} \\
<10 \mathrm{Mb} \\
\sim 25 \mathrm{Mb} \\
\sim 35 \mathrm{Mb}\end{array}$ \\
\hline
\end{tabular}


patient 1

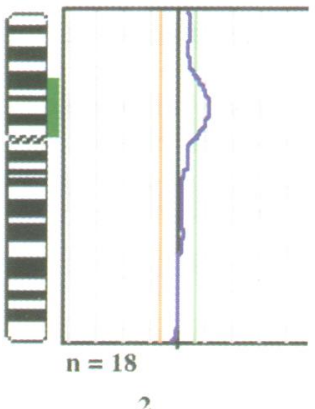

patient 2

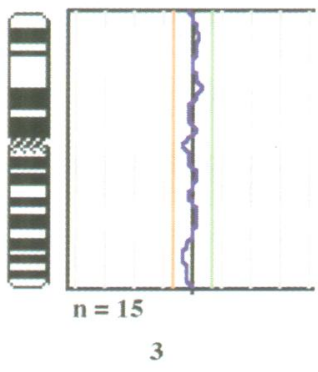

Chromosome specific CGH

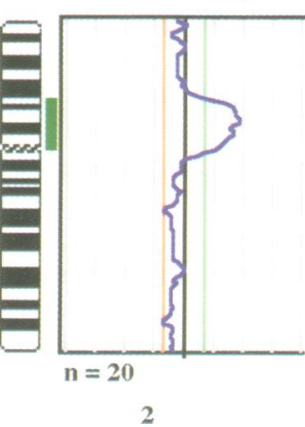

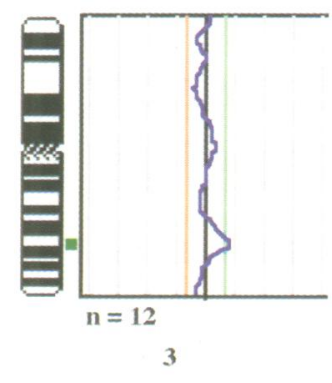

patient 3
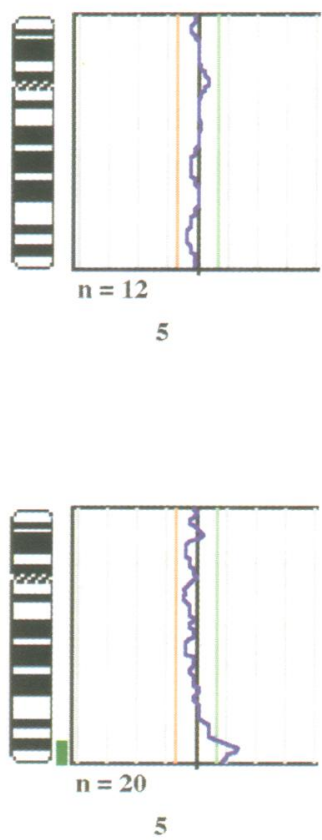

patient 4
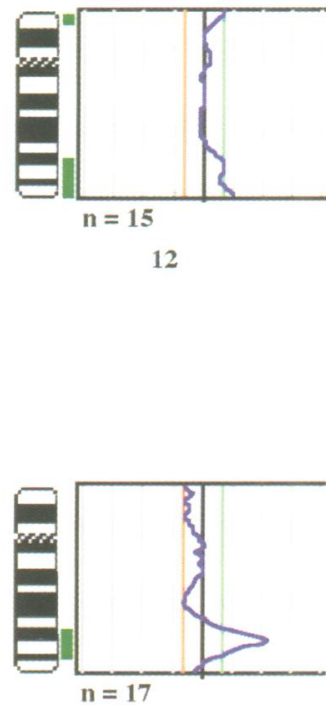

12

Figure 2 Ratio profiles of all patients comparing CGH with chromosome specific CGH.

Both for CGH and chromosome specific CGH experiments, Custom CGH software (Vysis "Quips" package) determined the chromosomal axis and measured the relative $\mathrm{red} / \mathrm{green}$ fluorescence ratio along it. In the case of chromosome specific $\mathrm{CGH}$, analysis was performed only on the chromosome pair on which hybridisation occurred by manually selecting only the two chromosomes of interest. The rest of the karyotype was thus disregarded. Following analysis of six to 10 metaphases, results were expressed as a ratio of red/green fluorescence and considered to represent a duplication when that ratio exceeded 1.15:1. In CGH experiments, ratios of between 1.15:1 and 1.25:1 are commonly used..$^{3-8}$ We chose the lower threshold to achieve maximum sensitivity when comparing CGH with chromosome specific CGH. Since fluorescent in situ hybridisation is not $100 \%$ efficient, the theoretical ratio of $1.5: 1$ is never reached.

\section{Results}

In each case, comparison with $\mathrm{G}$ banding data suggested that the duplication was tandem. This was detected for patients 1 and 4 using $\mathrm{CGH}$ and for all patients using chromosome specific $\mathrm{CGH}$; results are summarised in table 1. Fig 2 shows the $\mathrm{CGH}$ program profiles comparing the use of $\mathrm{CGH}$ with that of chromosome specific CGH. For patients 1 and 4 , the duplications on chromosomes 2 and 12 could be detected easily by both approaches although the peak for the chromosome specific CGH was higher and more clearly defined; indeed for patient 4 , it exceeds a ratio of $1.5: 1$, which is greater than can be theoretically achieved by CGH. Fig 3 shows that the smallest of duplications (for patient 2 on chromo- some 3) can be detected visually as an over-representation of red in the duplicated region. We were unable to detect the duplication in patients 2 and 3 using CGH; however, in both cases, we were successful using chromosome specific CGH. In patient 3, the duplication was at or near the end of the long arm of chromosome 5. The problems associated with detecting terminal duplications and deletions by CGH are well documented ${ }^{3}$; that is, CGH experiments commonly show apparent duplications or deletions at some telomeres which have subsequently been found not to be present; this seems to be the case for patient 4 where both telomeres show an apparent duplication. Conversely, duplications present at the termini of chromosomes can be missed by $\mathrm{CGH}$ and this seems to be the case for patient

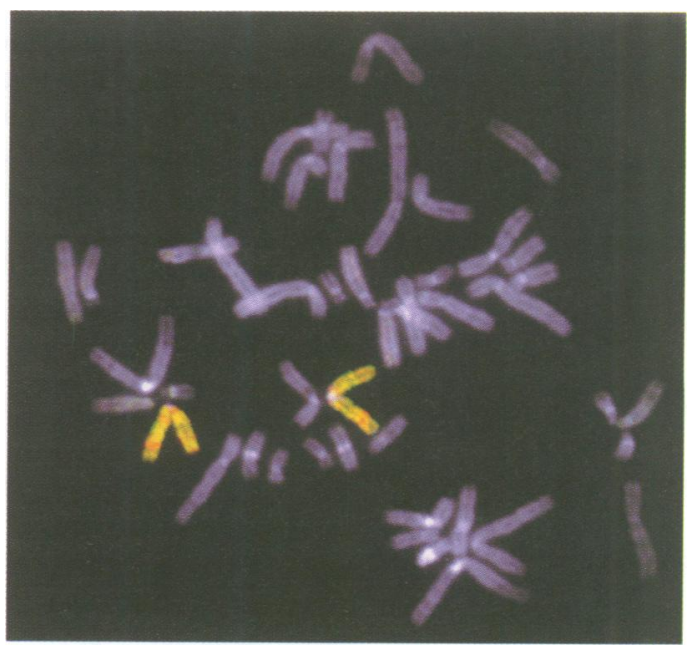

Figure 3 Fluorescent in situ hybridisation picture of patient 2. The over-representation of the red fluorochrome is clearly visible on the long arm of chromosome 3. 
3. In our limited experience of chromosome specific $\mathrm{CGH}$, a duplication at the terminus of chromosome 5 was easily detected and we have not yet seen an aberrant peak associated with the end of any chromosome.

\section{Discussion}

The use of the technique described here is limited to laboratories equipped for flow sorting; however, recovery of duplicated chromosomes could also be achieved by chromosome microdissection. ${ }^{4}$ Both approaches are laborious and require actively dividing samples to make metaphases and thus chromosome specific CGH is unlikely to be used as widely as conventional CGH. Nevertheless, our data show that chromosome specific $\mathrm{CGH}$ can detect duplications (and, presumably, amplifications or deletions) that may be missed by $\mathrm{CGH}$. This is because the ratio of hybridisation of abnormal/normal DNA in the duplicated region is $2: 1$. It is $3: 2$ in a $\mathrm{CGH}$ experiment owing to the presence of the normal homologue in the test hybridisation. $\mathrm{CGH}$ has been cited as a means of detecting constitutional duplications in patients where $G$ banding results are equivocal ${ }^{5}$; our results suggest that this new approach may be a more sensitive way of doing this, particularly where terminal duplications and deletions are concerned. It is unlikely that it would be used as a routine diagnostic tool, but it may prove invaluable in particular cases, for instance when the duplicated region contains a gene of interest. In the absence of molecular data, we cannot accurately determine the size of the duplications; further, we cannot rule out the possibility that other laboratories may be able to detect the smaller deletions by regular CGH. Indeed, several groups have reported the detection of aberrations as small as $10-20 \mathrm{Mb}$ by $\mathrm{CGH} .^{6-8}$ However, our data represent a set of controlled experiments where all other factors were kept equal other than the choice of probe; they clearly suggest that chromosome specific CGH is the more sensitive approach.

A more wide ranging application for this technology than the detection of duplications in patient material, however, is likely to be in the analysis of tumour cell lines. In addition to the added sensitivity provided by this approach over CGH, it has a number of other advantages. For instance, when analysing mosaic cell lines, that is, when duplicated chromosomes are present in only a small proportion of cells, duplications may be missed by $\mathrm{CGH}$ but detected by this method. This is also the case when there are more than two homologues, only one of which is duplicated. Further, it permits the use of longer chromosomes and hence more accurate band assignments, since locating metaphases without overlapping chromosomes is less problematical. That is, it is easier to locate metaphases where only one particular pair of homologues are not overlapping than divisions without a single chromosomal overlap. The purpose of CGH is to identify regions of interest, so that positional cloning of relevant genes may ensue; clearly an approach more sensitive than CGH for detect- ing such regions may find considerable applicability in showing amplified, duplicated, or deleted regions previously undiscovered.

The major technical drawback of this technique compared to $\mathrm{CGH}$ is the inherent necessity of previous flow sorting or chromosome microdissection. ${ }^{4}$ Thus it is limited to the analysis of actively growing cells from which chromosomes can be prepared; $\mathrm{CGH}$ on the other hand requires only a small amount of genomic DNA. Nevertheless, if these problems can be circumvented, a chromosome specific $\mathrm{CGH}$ experiment is much easier to perform than one involving regular $\mathrm{CGH}$ as analysis time is much shorter. This is because only one chromosome pair is analysed, the classification of chromosomes by DAPI banding is not necessary as the chromosome pair is clearly painted, and a number of experiments, each from a different patient sample, can be performed on one slide provided that there is a different chromosome of interest. Another potential drawback is the ability of flow sorting to differentiate the duplicated from the normal chromosome. The precise resolution power of flow sorting in this regard is unclear, but our results suggest that it is the hybridisation which is the rate limiting factor since, in patient 2 , the chromosomes were easily separated on the flow karyotype whereas the hybridisation ratio only just exceeded 1.15:1.

Finally, in this report we show that ratio analyses can be made from picogram quantities (that is, approximately 400 chromosomes) of DNA amplified by DOP-PCR. This may find applicability not only for chromosome specific $\mathrm{CGH}$ but for regular CGH also, for instance, in analysis of tumour progression from archive material, or for detecting aneuploidy diagnostically from small numbers of cells, perhaps for preimplantation diagnosis or in rare fetal cells isolated from the maternal circulation. Thus, we provide some evidence that DOP-PCR, in addition to being a powerful tool for amplifying small quantities of DNA, appears to amplify all or most of the euchromatic regions of the genome equally.

In summary, chromosome specific CGH uses the power of DOP-PCR to amplify small amounts of DNA equally and provides a sensitive means for the detection of intrachromosomal duplications. Although it has a number of (mostly technical) drawbacks compared to $\mathrm{CGH}$, we provide evidence that it has a greater sensitivity, particularly for the detection of terminal duplications. Furthermore, it clearly has an advantage over CGH in the ability to detect mosaic duplication and in situations when one of several homologues contains a duplication.

We would like to thank the staff of the Clinical Cytogenetic Laboratory, East Anglian Genetics Service, Addenbrooke's NHS Trust, Cambridge, UK for the provision of patient material and for supplying us with the cytogenetic reports. We are grateful to Professor Albert Schinzel for permission to use patient 2 in our study.

1 Carter NP, Ferguson-Smith MA, Perryman MT, et al. Reverse chromosome painting: a method for the rapid analysis of aberrant chromosomes in clinical cytogenetics. $\mathcal{F}$ analysis of aberrant chromosom

2 Telenius H, Pelmear AH, Tunnacliffe A, et al. Cytogenetic analysis by chromosome painting using DOP-PCR ampli- 
fied flow-sorted chromosomes. Genes Chrom Cancer 1992; 4:257-63.

3 Kallioniemi A, Kallioniemi OP, Sudar D, et al. Comparative genomic hybridization for molecular cytogenetic analysis of

Golid tumors. Science 1992;258:818-21.
Guan XY, Meltzer PS, Dalton WS, Trent JM. Identification of cryptic sites of DNA sequence amplification in human breast cancer by chromosome microdissection. Nat Genet

5 Bryndorf T, Kirchhoff M, Rose $\mathrm{H}$, et al. Comparative genomic hybridization in clinical cytogenetics. $\mathrm{Am} \mathcal{F} \mathrm{Hum}$ Genet 1995;57:1211-20.
6 Mannens M, Alders M, Redeker B, et al. Positional cloning of genes involved in the Beckwith-Wiedemann syndrome, hemihypertrophy, and associated childhood tumors. Med Pediatr Oncol 1996;27:490-4.

7 Wang BBT, Yu LC, Peng W, Falk RE, Williams J. Prenatal identification of $i(Y p)$ by molecular cytogenetic analysis. Prenat Diagn 1995;15:1115-19.

8 Weber RG, Sommer C, Albert FK, Kiessling M, Cremer T. Clinically distinct subgroups of glioblastoma-multiforme studied by comparative genomic hybridization. Lab Invest 1996;74:108-19. 